\title{
Vitesses de réaction de dissolution et précipitation au voisinage de l'interface oxydo-réducteur dans un lac méromictique : le lac Pavin (Puy de Dôme, France) \\ Dissolution and precipitation reactions at the redox interface in a meromictic lake (Pavin lake, France)
}

\author{
G. Michard, D. Jezequel et E. Viollier
}

Volume 16, numéro 2, 2003

URI : https://id.erudit.org/iderudit/705504ar

DOI : https://doi.org/10.7202/705504ar

\section{Aller au sommaire du numéro}

\section{Éditeur(s)}

Université du Québec - INRS-Eau, Terre et Environnement (INRS-ETE)

ISSN

0992-7158 (imprimé)

1718-8598 (numérique)

\section{Découvrir la revue}

\section{Citer cet article}

Michard, G., Jezequel, D. \& Viollier, E. (2003). Vitesses de réaction de dissolution et précipitation au voisinage de l'interface oxydo-réducteur dans un lac méromictique : le lac Pavin (Puy de Dôme, France). Revue des sciences de l'eau / Journal of Water Science, 16(2), 199-218. https://doi.org/10.7202/705504ar

\section{Résumé de l'article}

Une étude à l'échelle centimétrique de l'interface redox situé à la limite entre mixolimnion et monimolimnion d'un lac méromictique (le lac Pavin) a permis d'observer très finement l'évolution de la concentration d'un certain nombre d'éléments chimiques. Nous avons choisi de présenter ici des résultats concernant 5 éléments qui présentent des comportements très contrastés : le rubidium, le fer, le baryum, le vanadium et le manganèse. La comparaison avec un élément conservatif, le sodium, montre que $\mathrm{Rb}$ est conservatif, que Fe, $\mathrm{Ba}$ et $\mathrm{V}$ sont précipités et que $\mathrm{Mn}$ est dissous dans cette zone.

Une modélisation de ces concentrations en vue de préciser à quelle profondeur et avec quelle vitesse se produisent les réactions concernant ces éléments nécessite la détermination des paramètres de transport au voisinage de cet interface.

Une représentation analytique des concentrations de sodium permet de calculer le coefficient de diffusion turbulente $\mathrm{K}_{\mathrm{z}}$ en fonction de la profondeur. $\mathrm{Au}$ voisinage de l'interface redox, ce coefficient est très petit $\left(0,0017 \mathrm{~m}^{2} /\right.$ jour $)$ et inférieur au coefficient de diffusion thermique moléculaire.

Les concentrations des éléments étudiés ont pu être représentés avec précisions par des polynômes en fonction de la concentration en sodium.

Cela permet d'estimer les vitesses des réactions de précipitation dissolution en fonction de la profondeur. Le rubidium n'est affecté par aucune réaction. Le fer précipite entre 63 et $65 \mathrm{~m}$, le baryum entre 68 et $72 \mathrm{~m}$ tandis que le vanadium précipite à la fois dans ces 2 zones. Le manganèse réagit dans une zone très étroite : il est précipité entre 61,5 et $62 \mathrm{~m}$ et dissous entre 62,8 et 63,1 $\mathrm{m}$.

Une étude similaire de tous les éléments majeurs (y compris pH et COD) pourrait permettre d'élucider les processus qui conduisent à ces comportements complexes. 


\title{
Vitesses des réactions de dissolution et précipitation au voisinage de l'interface oxydo-réducteur dans un lac méromictique : le lac Pavin (Puy de Dôme, France)
}

\author{
Dissolution and precipitation reactions at the redox \\ interface in a meromictic lake (Pavin lake, France)
}

\section{G. MICHARD*, D. JEZEQUEL et É. VIOLLIER}

Reçu le 7 mai 2002, accepté le 10 décembre 2002**.

\section{SUMMARY}

Lake Pavin, French Massif Central, is the main meromictic lake in France and has been extensively studied from more than 50 years. The upper part (mixolimnion) at a depth of less than about $60 \mathrm{~m}$ behaves as an oligotrophic lake and is oxic during the major part of the year. The lower layer (monimolimnion) has a higher salinity and is permanently anoxic. Unlike the top of the mixolimnion, element concentrations in the monimolimnion can be considered at steady state. The boundary between mixolimnion and monimolimnion is a redox interface. At this interface, an important number of both chemical and biochemical reactions occur.

This boundary, where element concentrations vary greatly, was studied at the centimeter scale between 58 and $64 \mathrm{~m}$ depth. The present paper is focused on five elements showing very different behaviours: rubidium, iron, manganese, vanadium and barium. Sodium was used as a reference element. Sodium and rubidium concentrations had similar patterns: a progressive increase began at $61 \mathrm{~m}$ depth and the maximal gradient was located at $63 \mathrm{~m}$. They continue to increase towards the bottom of the lake. Iron concentrations were low $(<1 \mu \mathrm{mol} / \mathrm{L})$ at a depth less than $62.8 \mathrm{~m}$ and increased very sharply below this depth. Manganese concentrations were very low in the mixolimnion $(<0.01 \mu \mathrm{mol} / \mathrm{L})$, exhibited a peak between 62.4 and $63.5 \mathrm{~m}$ depth (up to $60 \mu \mathrm{mol} / \mathrm{L}$ at $63 \mathrm{~m}$ ) and reached a value of about $30 \mu \mathrm{mol} / \mathrm{L}$ at $85 \mathrm{~m}$. Barium concentrations began to increase only at depths greater than 65-67 m. Vanadium concentrations in the mixolimnion were about $14 \mathrm{nmol} / \mathrm{L}$, decreased to a minimum below the detection limit at $62.2 \mathrm{~m}$ and then increase drastically $(150 \mathrm{nmol} / \mathrm{L}$ at $85 \mathrm{~m})$.

In order to derive the accurate location of the chemical reactions and an estimation of their rates from the concentration profiles, knowledge of the trans-

Laboratoire de Géochimie des Eaux, Université Paris 7 et IPGParis, Tour 53/54, 2, place Jussieu, 75251 Paris cedex 05, France.

* Correspondance. E-mail : michargf@noos.fr

** Les commentaires seront reçus jusqu'au 30 décembre 2003. 
port parameters was needed. As advection can be considered to be negligible, the major parameter of interest is the vertical eddy diffusion coefficient $K_{z}$. $\mathrm{Na}$ is assumed to be unreactive in the studied layer. Its concentrations can be represented by an analytical function

$$
\mathrm{C}=\frac{C_{\max }-C_{\min }}{2} * \operatorname{th}[P(z)]+\frac{C_{\max }+C_{\min }}{2}
$$

with $P(z)=0.0016 *\left(z-z_{0}\right)^{3}-0.0493 *\left(z-z_{0}\right)^{2}+0.5735 *\left(z-z_{0}\right)-0.4811$

This allows the determination of the coefficient $K_{z}$.

$$
\mathbf{K}_{\mathbf{z}}=\lambda \operatorname{ch}^{2}[\mathbf{P}(\mathbf{z})] /\left[\mathbf{P}^{\prime}(\mathbf{z})\right]
$$

$\lambda$ is determined from the value of $K_{z}$ at $85 \mathrm{~m}$ depth previously obtained from an hydrodynamic study of the lake (AESHBACH-HERTIG $e t$ al., 1999). This coefficient is about $0.1 \mathrm{~m}^{2} / \mathrm{day}$ at the bottom of the monimolimnion. It is very low at the redox interface $\left(0.0017 \mathrm{~m}^{2} /\right.$ day), far below the molecular thermal diffusion coefficient. It increases very sharply at the bottom of the mixolimnion. The $K_{z}$ profile is in fair agreement with the results obtained from the earlier hydrodynamic study.

A quantitative study of the dissolution-precipitation reactions at the center of the lake at depths between 55 and $85 \mathrm{~m}$ can then be undertaken. The $55 \mathrm{~m}$ limit corresponds to a depth where inputs of fresh water can occur. The $85 \mathrm{~m}$ limit is about $7 \mathrm{~m}$ above the bottom of the lake. Below this depth important inputs from the pore waters occur which are not taken into account by the present modeling. Concentrations of $\mathrm{Rb}, \mathrm{Fe}, \mathrm{Ba}$ and $\mathrm{V}$ can be accurately represented by polynomial functions of the $\mathrm{Na}$ concentration. The parameter $u=\operatorname{th}[P(z)]$ represents the concentrations of these 4 elements by polynomials :

$$
X(u)=\sum_{0}^{N} a_{n} \cdot u^{n}
$$

The rate of dissolution-precipitation for each element as a function of depth can be derived.

$$
\mathbf{R}=-\lambda\left[\mathbf{P}^{\prime}(\mathbf{z})\right] * \operatorname{ch}^{-2}[\mathbf{P}(\mathbf{z})] \sum_{0}^{N} \mathbf{a}_{\mathbf{n}} \mathbf{n}(n-1) * \operatorname{th}^{n-2}[\mathbf{P}(\mathbf{z})]
$$

$\mathrm{Rb}$ concentrations are a linear function of the Na ones and therefore rubidium is not reactive. Fe concentrations can be related to sodium concentrations by a parabolic relationship. From this relationship, it can be derived that strong iron precipitation occurs in the $63-65 \mathrm{~m}$ depth layer. $V$ concentrations are related to sodium ones by a $4^{\text {th }}$ degree polynomial. It can be derived that $V$ deposition occurs at depths of 63-65 $\mathrm{m}$ and at $70 \mathrm{~m}$.

Ba precipitates around $70 \mathrm{~m}$ depth. Mn concentrations are represented by

$$
[M n]=a_{0}+a_{1} u+b_{1} \exp \left[-\left(z-z^{\circ}\right)^{2} / z^{* 2}\right]
$$

and the derivation shows that $M n$ is strongly dissolved between 62.8 and $63 \mathrm{~m}$ and precipitated just above. These results are in good agreement with a previous study of particles fluxes derived from sediment trap analysis (VIOLLIER et al., 1997).

This study shows the complexity of this interface and more comprehensive studies including all major elements, dissolved organic carbon (DOC) and pH are needed. 


\section{RÉSUMÉ}

Une étude à l'échelle centimétrique de l'interface redox situé à la limite entre mixolimnion et monimolimnion d'un lac méromictique (le lac Pavin) a permis d'observer très finement l'évolution de la concentration d'un certain nombre d'éléments chimiques. Nous avons choisi de présenter ici des résultats concernant 5 éléments qui présentent des comportements très contrastés : le rubidium, le fer, le baryum, le vanadium et le manganèse. La comparaison avec un élément conservatif, le sodium, montre que $\mathrm{Rb}$ est conservatif, que $\mathrm{Fe}, \mathrm{Ba}$ et V sont précipités et que Mn est dissous dans cette zone.

Une modélisation de ces concentrations en vue de préciser à quelle profondeur et avec quelle vitesse se produisent les réactions concernant ces éléments nécessite la détermination des paramètres de transport au voisinage de cet interface.

Une représentation analytique des concentrations de sodium permet de calculer le coefficient de diffusion turbulente $K_{z}$ en fonction de la profondeur. Au voisinage de l'interface redox, ce coefficient est très petit $\left(0,0017 \mathrm{~m}^{2} /\right.$ jour $)$ et inférieur au coefficient de diffusion thermique moléculaire.

Les concentrations des éléments étudiés ont pu être représentées avec précisions par des polynômes en fonction de la concentration en sodium.

Cela permet d'estimer les vitesses des réactions de précipitation dissolution en fonction de la profondeur. Le rubidium n'est affecté par aucune réaction. Le fer précipite entre 63 et $65 \mathrm{~m}$, le baryum entre 68 et $72 \mathrm{~m}$ tandis que le vanadium précipite à la fois dans ces 2 zones. Le manganèse réagit dans une zone très étroite : il est précipité entre 61,5 et $62 \mathrm{~m}$ et dissous entre 62,8 et $63,1 \mathrm{~m}$.

Une étude similaire de tous les éléments majeurs (y compris pH et COD) pourrait permettre d'élucider les processus qui conduisent à ces comportements complexes.

\section{1 - INTRODUCTION}

Les lacs constituent de gigantesques réacteurs chimiques (Gaillard, 1995). Le couplage de la stratification thermique et des réactions de photosynthèse respiration conduisent à des variations spatiales et temporelles des concentrations des espèces dissoutes. Ces variations concernent en premier lieu les nutriments (cf. WETZEL, 1983 ;.GAILLARD, 1995 ; MICHARD, 2002), les éléments dont la solubilité est liée aux conditions redox, comme le fer et le manganèse (MORTIMER, 1941 ; DAVISON, 1993 ; HAMILTON-TAYLOR and DAVISON, 1995) et aussi des éléments trace comme le cobalt (BALISTRIERI et al., 1992, 1994 ; TAILLEFERT et al., 2002), le baryum (SUGIYAMA et al., 1992 ; VIOLLIER, 1995) ou le vanadium (Viollier et al., 1995).

Si des variations de concentrations ont été décrites dans de nombreux articles, leur compréhension quantitative est encore abordée de façon très fragmentaire. A l'inverse, les eaux interstitielles des sédiments, dans lesquelles se produisent des réactions analogues ont fait depuis longtemps l'objet de modélisation concernant d'abord un composé, comme les sulfates (BERNER, 1964) ou le manganèse (MICHARD, 1971), puis l'ensemble des réactions qui s'y 
produisent (RABOUILLE et GAILLARD, 1991 ; VAN CAPPELLEN et WANG, 1996 ; WANG et VAN CAPPELLEN, 1997). Toutes ces modélisations sont effectuées à l'état stationnaire et les processus de transport se réduisent à la diffusion moléculaire (plus ou moins ralentie par les effets de tortuosité et l'adsorption des éléments à la surface des particules) et à l'advection liée à la vitesse de sédimentation. Les difficultés de la modélisation de la colonne d'eau d'un lac tiennent d'une part aux importantes variations temporelles des grandeurs à modéliser, d'autre part à la difficulté de connaître les paramètres de transport, car la diffusion y est turbulente (IMBODEN and WUEST, 1995).

Dans cet article, nous nous limiterons à une étude à l'état stationnaire d'une des rares zones d'un système lacustre à laquelle on peut envisager d'appliquer cette hypothèse simplificatrice : le monimolimnion d'un lac méromictique. Dans un lac suffisamment creux (dont le paramètre sans dimension creux = profondeur maximale divisée par la racine carrée de la surface du lac est supérieur à 0,1 ), le mélange des eaux qui se produit à l'automne et éventuellement au printemps, quand la température du lac est uniforme, ne concerne pas les couches les plus profondes. Elles forment alors une couche stable appelée monimolimnion ; le lac est alors qualifié de méromictique (DUSSART, 1966).

La deuxième difficulté, la détermination des coefficients de diffusion turbulente, sera par contre un problème à résoudre.

L'exemple que nous présentons ici est relatif au principal lac méromictique français, le lac Pavin, qui a fait l'objet de nombreuses études (BERTHOULE, 1896 ; DELEBECQUE, 1898 ; OLIVIER, 1952 ; ALVINERIE et al., 1966 ; PELLETIER, 1968 ; MEYBECK et al., 1975 ; DEVAUX et al., 1983 ; AMBLARD et RESTITUITO, 1984 ; RESTITUITO, 1984, 1987 ; MARTIN, 1985 ; MARTIN et al., 1992 ; CAMUS et al., 1993 ; COSSA et al., 1994 ; MICHARD et al.,1994 ; VIOLLIER et al., 1995, 1997 ; AESCHBACH-HERTIG et al., 1999 ; ALBÉRIC et al., 2000). II ne s'agit pas, comme dans les modélisations de la chimie des eaux interstitielles citées précédemment, de prévoir la composition chimique à partir d'une série de réactions posées a priori, mais d'obtenir à partir des concentrations observées des informations sur les réactions faisant passer les éléments de la phase dissoute à la phase solide ou inversement. Nous appellerons par la suite ces réactions de précipitation ou de dissolution, bien que par exemple les premières, outre les réactions de précipitation proprement dites, incluent aussi la consommation par les organismes et les adsorptions à la surface de solides.

\section{2 - SITE D’ÉTUDE ET MÉTHODOLOGIE}

Le lac Pavin est un lac de cratère situé par $45^{\circ} 55^{\prime} \mathrm{N}$ et $2^{\circ} 54^{\prime} \mathrm{E}$, à l'écart de toute ville et de tout centre industriel importants. L'altitude de la surface est de $1197 \mathrm{~m}$. II est de forme circulaire de $750 \mathrm{~m}$ de diamètre et sa profondeur maximale est de $92 \mathrm{~m}$ (DELEBECQUE, 1898). L'aire du bassin de drainage est de $0,8 \mathrm{~km}^{2}$.

Il est divisé en 2 couches:

- le mixolimnion qui est affecté par le mélange d'hiver et qui se comporte comme un lac oligotrophe avec épilimnion et hypolimnion en été où l'oxygène reste présent pendant une bonne partie de l'année, 
- le monimolimnion aux profondeurs supérieures à 60-62 m qui est stable et anoxique en permanence.

Les échantillons ont été prélevés en juin 1998 tous les 5 ou $10 \mathrm{~m}$ de profondeur au voisinage du centre du lac par une seringue dont le déclenchement est assuré par l'envoi d'un messager. Pour mieux cerner les variations au voisinage de l'interface mixolimnion - monimolimnion (IMM), des prélèvements spécifiques ont été faits en utilisant des regards à sédiments ou " peepers » (Hesslein, 1976). Ordinairement utilisés pour le prélèvement des eaux interstitielles, ces outils se sont révélés très précieux pour l'analyse à l'échelle de quelques $\mathrm{cm}$ de l'IMM. Des études préliminaires ont montré que dans une eau libre, le temps d'équilibration était inférieur à 24 heures. Cinq peepers, dont les loges sont distantes de $2,5 \mathrm{~cm}$ environ, ont été mis bout à bout entre les profondeurs de 58,12 et $63,50 \mathrm{~m}$ le long d'un câble de nylon tendu entre un corps mort et une bouée de subsurface. L'ensemble a été maintenu en place pendant 48 heures. Les peepers sont alors retirés, rapidement stockés dans des sacs de polyéthylène gonflés à l'azote et le prélèvement du contenu de chacune des loges se fait en piquant, à la fois à travers le sac et la membrane de la loge, l'aiguille d'un dispositif analogue à celui que l'on utilise pour les prélèvements sanguins. Dans une première étape, les échantillons ont été stockés après acidification (avec $\mathrm{HNO}_{3}$ de haute pureté) et analysés au laboratoire.

La précision sur la profondeur des prélèvements avec la seringue est de l'ordre de 1 à $2 \%$; elle est liée à un défaut possible de verticalité du câble. Les différents prélèvements correspondent à des descentes différentes du dispositif et les erreurs sont indépendantes. Par contre, l'incertitude est beaucoup plus faible sur les prélèvements par peeper, car le câble est maintenu en position fixe et surtout l'intervalle entre 2 profondeurs de prélèvement reste fixe puisqu'il correspond à l'intervalle entre 2 loges du peeper.

L'inconvénient de ce procédé est que l'on obtient simultanément une centaine d'échantillons sur lesquels il faudrait, outre le traitement par acidification, effectuer les mesures immédiates des composés instables à l'air : sulfure, phosphate, ammonium, et alcalinité.

C'est pourquoi, dans ce premier temps, nous ne nous sommes intéressés qu'à des éléments dont le traitement par acidification préserve les concentrations. Dans cet article, nous présentons les résultats concernant 5 éléments dont le comportement est différent: $\mathrm{Rb}, \mathrm{Fe}, \mathrm{Ba}, \mathrm{V}$, et $\mathrm{Mn}$. Le sodium a été choisi comme élément de référence.

Les analyses ont été effectuées par spectrophotométrie d'absorption atomique, en flamme pour les éléments alcalins et alcalino terreux majeurs, en four graphite pour Fe et Mn. Les éléments trace ont été analysés par ICP-MS au Laboratoire Pierre Süe à Saclay. Les résultats obtenus sur les standards internationaux ont été déjà publiés (VIOLLIER et al., 1995).

\section{3 - RÉSULTATS}

Les données analytiques sont regroupées dans le tableau 1. 
Tableau 1 Concentrations de $\mathrm{Na}, \mathrm{Rb}, \mathrm{Fe}, \mathrm{V}, \mathrm{Ba}$ et $\mathrm{Mn}$ en fonction de la profondeur. Table 1 $\mathrm{Na}, \mathrm{Rb}, \mathrm{Fe}, \mathrm{V}, \mathrm{Ba}$ and $\mathrm{Mn}$ concentrations versus depth.

\begin{tabular}{ccccccc}
\hline $\begin{array}{c}\text { Profondeur } \\
(\mathbf{m})\end{array}$ & $\begin{array}{c}\mathbf{N a} \\
\left(\mathbf{m m o l} / \mathbf{m}^{3}\right)\end{array}$ & $\begin{array}{c}\mathbf{F e} \\
\left(\mathbf{m m o l} / \mathbf{m}^{\mathbf{3}}\right)\end{array}$ & $\begin{array}{c}\mathbf{M n} \\
\left(\mathbf{m m o l} / \mathbf{m}^{\mathbf{3}}\right)\end{array}$ & $\begin{array}{c}\mathbf{V} \\
\left(\mu \mathbf{m o l} / \mathbf{m}^{\mathbf{3}}\right)\end{array}$ & $\begin{array}{c}\mathbf{B a} \\
\left(\mu \mathbf{m o l} / \mathbf{m}^{3}\right)\end{array}$ & $\begin{array}{c}\mathbf{R b} \\
\left(\mu \mathbf{m o l} / \mathbf{m}^{\mathbf{3}}\right)\end{array}$ \\
\hline 63,19 & 309 & & & 20,02 & 14,4 & 144 \\
63,22 & 311 & & 50,4 & 20,96 & 14,3 & 146 \\
63,50 & & 200 & 35,6 & 46,10 & 10,2 & \\
65 & 384 & 580 & 28,4 & 47,0 & 10,3 & 192 \\
67,5 & & 700 & 28,6 & 71,5 & 30 & \\
70 & 430 & 837 & 30,5 & 97,1 & 96 & 234 \\
75 & 456 & 996 & 34 & 123,9 & 264 & 253 \\
80 & 463 & 1084 & 35,9 & 144,9 & 525 & 253 \\
85 & 469 & 1154 & 35,2 & 154,2 & 718 & 260 \\
87 & 474 & 1184 & & & 841 & 266 \\
\hline
\end{tabular}

Les concentrations des six éléments sont nettement plus élevées dans le monimolimnion, au moins à sa partie inférieure, que dans le mixolimnion. Cela est dû en grande partie aux réactions qui se produisent au fond du lac et à un degré moindre à l'apport d'une source minérale de très faible débit (VIOLLIER et al., 1997 ; MiCHARD et al., 2002). Mais la façon dont les concentrations augmentent au voisinage de l'IMM est très variable entre les 6 éléments.

Les concentrations de rubidium, comme celles de sodium, commencent à augmenter à la profondeur de $61 \mathrm{~m}$; la croissance est de plus en plus forte jusqu'à $63 \mathrm{~m}$, puis elle diminue tout en restant positive.

Les teneurs en fer sont faibles $(<0,1 \mu \mathrm{mol} / L)$ aux profondeurs inférieures à $62,8 \mathrm{~m}$; puis elle croissent très rapidement : $49 \mu \mathrm{mol} / \mathrm{L}$ dix $\mathrm{cm}$ plus bas et $85 \mu \mathrm{mol} / \mathrm{L} 23 \mathrm{~cm}$ plus bas $(63,03 \mathrm{~m})$ pour atteindre $1150 \mu \mathrm{mol} / \mathrm{L}$ à $85 \mathrm{~m}$.

Les concentrations en manganèse sont faibles $(<0,01 \mu \mathrm{mol} / L)$ jusqu'à $59 \mathrm{~m}$ puis croissent faiblement de $59,4 \mathrm{~m}(0,007 \mu \mathrm{mol} / \mathrm{L})$ à $61,0 \mathrm{~m}(0,1 \mu \mathrm{mol} / \mathrm{L})$, puis très vite en passant par un maximum très aigu $(30 \mu \mathrm{mol} / \mathrm{L}$ à $62,36 \mathrm{~m}$; $60,4 \mu \mathrm{mol} / \mathrm{L}$ à $63,03 \mathrm{~m} ; 35,6 \mu \mathrm{mol} / \mathrm{L}$ à $63,5 \mathrm{~m}$ ) décroissent jusqu'à $28 \mu \mathrm{mol} / \mathrm{L}$ (65-67,5 $\mathrm{m}$ de profondeur) pour remonter à $35 \mu \mathrm{mol} / \mathrm{L}$ à $85 \mathrm{~m}$.

Les teneurs en baryum dans le mixolimnion sont en moyenne de $9 \mathrm{nmol} / \mathrm{L}$. Elles oscillent entre 8 et $15 \mathrm{nmol} / \mathrm{L}$ jusqu'à la profondeur de $65 \mathrm{~m}$ et croissent très fortement en dessous $(30 \mathrm{nmol} / \mathrm{L}$ à $67,5 \mathrm{~m}$ et $718 \mathrm{nmol} / \mathrm{L}$ à $85 \mathrm{~m})$.

Les concentrations en vanadium sont en moyenne de $14 \mathrm{nmol} / \mathrm{L}$ dans le mixolimnion. Elles décroissent et passent par un minimum inférieur à la limite de détection $(0,2 \mathrm{nmol} / \mathrm{L})$ à $62,2 \mathrm{~m}$, et croissent rapidement en dessous $(8 \mathrm{nmol} / \mathrm{L}$ à $63 \mathrm{~m} ; 150 \mathrm{nmol} / \mathrm{L}$ à $85 \mathrm{~m})$.

\section{4 - DISCUSSION}

Dans des discussions précédentes (en particulier Michard et al., 1994 et VIOLLIER et al., 1995), des corrélations entre concentrations d'éléments avaient 
permis de distinguer entre les éléments non réactifs, liés entre eux par des corrélations linéaires, que l'on avait appelés éléments conservatifs et les éléments « réactifs". Les éléments conservatifs ne subissent aucune réaction de dissolution - précipitation dans la zone considérée, ce qui n'est certainement pas le cas au fond du lac où des réactions importantes se produisent. Ces éléments sont par exemple $\mathrm{Na}, \mathrm{K}, \mathrm{Cl}, \mathrm{Mg}$, $\mathrm{Li}, \mathrm{Rb}$, etc.

Les concentrations des éléments réactifs présentent des corrélations non linéaires avec la concentration d'un conservatif : au-dessous de la droite de mélange si l'élément est précipité, au-dessus s'il subit des dissolutions. Ce sont ces réactions dont nous voulons préciser la localisation et l'intensité.

Pour cela, nous allons utiliser le profil d'un élément conservatif pour contraindre le coefficient de diffusion turbulente verticale $\mathrm{K}_{\mathrm{z}}$ en fonction de la profondeur et utiliser ce résultat pour déterminer en fonction de la profondeur la vitesse de la réaction de dissolution - précipitation pour chaque élément réactif.

\subsection{Détermination du coefficient de diffusion turbulente verticale $K_{z}$}

Dans un lac, les processus de transport sont beaucoup plus lents dans le sens vertical et les concentrations dans un plan horizontal sont sensiblement constantes sauf au proche voisinage des bords (BOYCE and HAMBLIN, 1975) et les concentrations sont constantes dans un plan horizontal. L'invariance de la température et des concentrations dans le plan horizontal a été vérifiée dans le lac Pavin (MICHARD et al., 2002). On peut donc traiter le problème à 1 dimension. Pour ne pas tenir compte des effets de la berge, nous limiterons la modélisation à des profondeurs $(<85 \mathrm{~m}$ ) auxquelles les dimensions horizontales sont importantes (200 m de diamètre). Au centre du lac, la variation de concentration d'un élément à la cote $z$ et au temps $t$ s'écrit :

$$
\frac{\partial \mathrm{C}}{\partial \mathrm{t}}=\frac{\partial}{\partial z}\left(\mathrm{~K}_{\mathrm{z}} \cdot \frac{\partial \mathrm{C}}{\partial \mathrm{z}}\right)-\omega \frac{\partial \mathrm{C}}{\partial \mathrm{z}}+\mathrm{R}
$$

où $\omega$ désigne la vitesse d'advection, $\mathrm{R}$ le terme de réaction chimique à la cote $z$.

Pour un élément conservatif, $\mathrm{R}=0$.

Dans le lac Pavin, w est très petit, voire nul (Aeschbach-Hertig et al., 2002). En outre, ces auteurs montrent que l'on peut considérer que la composition du lac peut être considérée comme étant à l'état stationnaire.

Pour un élément conservatif, on peut donc réécrire l'équation (1) :

$$
\frac{\partial \mathrm{C}}{\partial \mathrm{t}}=\frac{\partial}{\partial \mathrm{z}}\left(\mathrm{K}_{\mathrm{z}} \cdot \frac{\partial \mathrm{C}}{\partial \mathrm{z}}\right)=0
$$

soit :

$$
\mathrm{K}_{\mathrm{z}} \cdot \frac{\partial \mathrm{C}}{\partial \mathrm{z}}=\text { constante }=\alpha
$$




\subsubsection{Représentation analytique de la concentration d'un élément conservatif}

L'utilisation de la relation (2) nécessite la représentation de la concentration $\mathrm{C}$ d'un élément conservatif par une fonction continue et dérivable.

Posons

$$
\mathrm{u}=\frac{\mathrm{C}-\frac{\mathrm{C}_{\text {max }}+\mathrm{C}_{\text {min }}}{2}}{\frac{\mathrm{C}_{\max }-\mathrm{C}_{\min }}{2}}
$$

où $\mathrm{C}_{\min }$ et $\mathrm{C}_{\max }$ représentent respectivement la concentration minimale et la concentration maximale de l'élément conservatif.

$$
-1 \leq u \leq 1
$$

En portant $u$ en fonction de $z$, on obtient une courbe assez similaire à

th $\left(z-z_{0}\right)$. On peut améliorer la représentation en portant la fonction inverse Argth(u) en fonction de $z$. On obtient alors une série de points auxquels on peut ajuster un polynôme en $z$, soit $P(z)$.

Nous choisirons le sodium comme élément conservatif type. La figure 1a montre comment $P(z)$ peut être obtenu (en posant $z^{\prime}=z-z_{0}$, avec $z_{0}=63,5 \mathrm{~m}$,

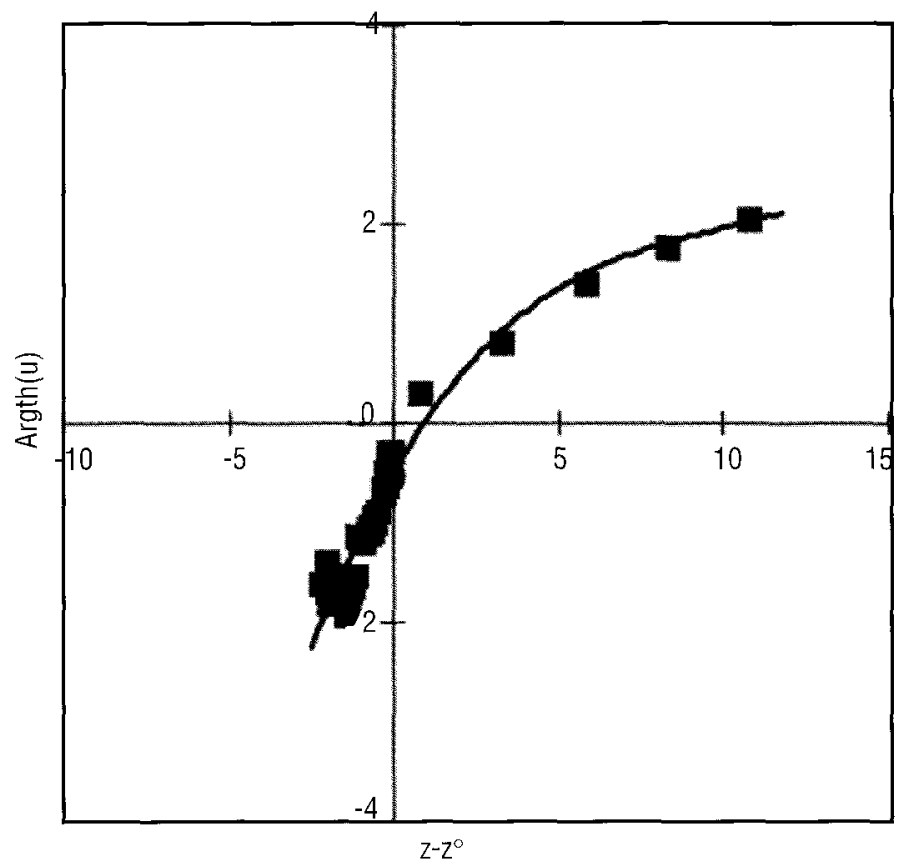

Figure 1a Ajustement de Argth $(u)$ en fonction de la profondeur ; $z^{\circ}=63,5 \mathrm{~m}$. Fit of Argth $(u)$ versus depth; $z^{\circ}=63.5 \mathrm{~m}$. 
valeur qui donne pour la relation polynomiale le coefficient de corrélation le plus élevé $\left(R^{2}=0,96\right)$.

$$
\text { Argth }(u) P(z)=0,0016 \cdot z^{\prime 3}-0,0493 \cdot z^{\prime 2}+0,5735 \cdot z^{\prime}-0,4811
$$

Le relation (3) conduit à

$$
\begin{gathered}
C=\frac{C_{\max }-C_{\min }}{2} \cdot u+\frac{C_{\max }+C_{\min }}{2} \\
C=\frac{C_{\text {max }}-C_{\min }}{2} \cdot \operatorname{th}[P(z)]+\frac{C_{\text {max }}+C_{\min }}{2}
\end{gathered}
$$

La figure 1b démontre que la fonction proposée représente correctement les observations. Le seul point qui n'est pas sur la courbe est celui qui correspond à la profondeur de $65 \mathrm{~m}$. Comme nous l'avons mentionné plus haut, l'incertitude sur la différence de profondeur entre ce point et la base du peeper le plus profond est maximale et peut atteindre $1 \mathrm{~m}$.

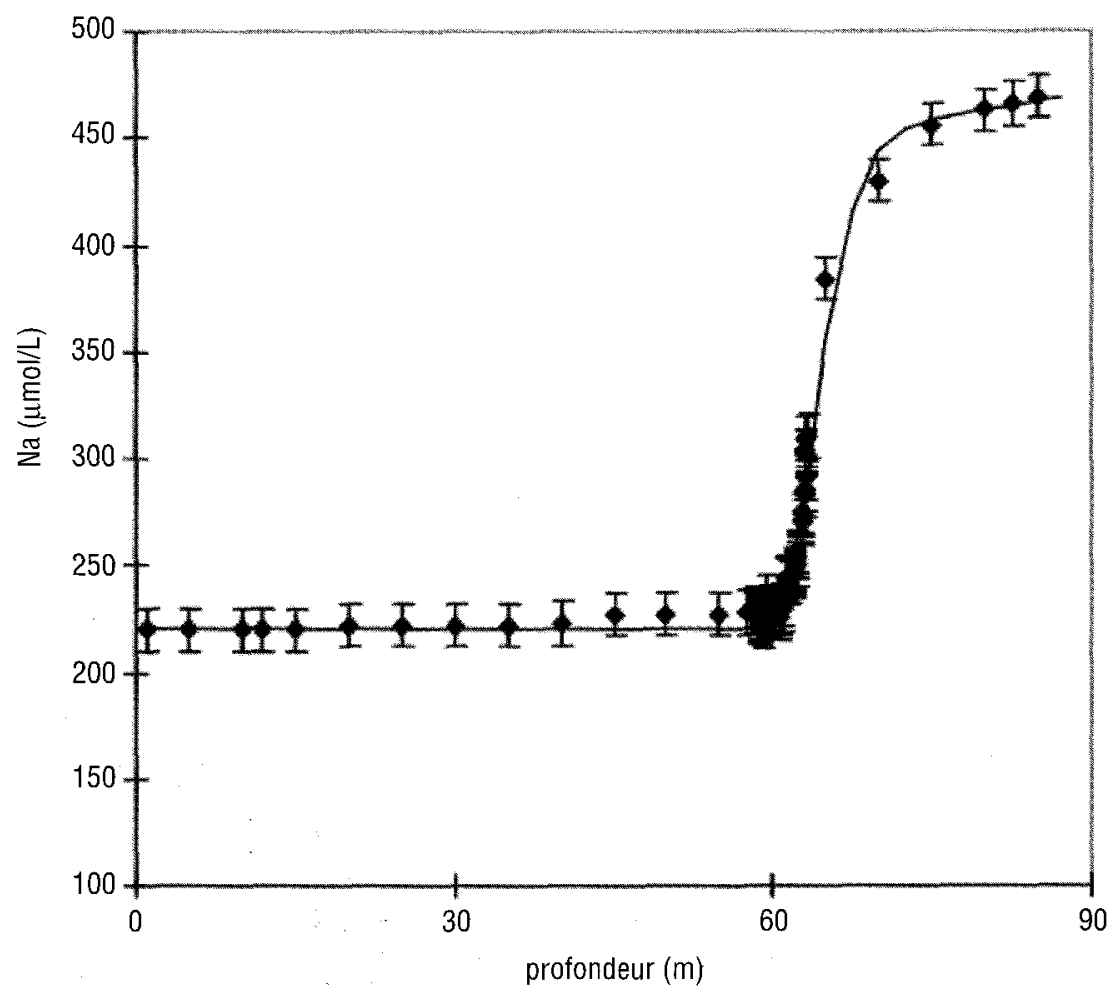

Figure 1b Concentration du sodium dans le lac Pavin en fonction de la profondeur. Les losanges représentent les valeurs observées ; la courbe en noir est la fonction définie par l'équation (3).

Sodium concentration in lake Pavin versus depth. Diamonds are the observed concentrations; the black curve is the function defined by equation (3). 
Dans la suite, pour alléger l'écriture, nous écrirons:

$\mathrm{C}=\mathrm{A} \cdot \operatorname{th}[\mathrm{P}(\mathrm{z})]+\mathrm{B}$

\subsubsection{Calcul du coefficient de diffusion turbulente verticale}

II suffit de reporter les expressions des dérivées première et seconde de (4') dans l'équation (2)

$$
d C / d z=A \cdot P^{\prime}(z) \cdot c h^{-2}[P(z)]
$$

soit en reportant dans (2) et en posant $\lambda=\alpha / \mathrm{A}$

$$
\mathrm{K}_{\mathrm{z}}=\lambda \mathrm{ch}^{2}[\mathrm{P}(\mathrm{z})] /\left[\mathrm{P}^{\prime}(\mathrm{z})\right]
$$

$\lambda$ étant déterminé par une valeur particulière de $\mathrm{K}_{\mathrm{z}}$.

Aeschbach-Hertig et al. (2002) proposent pour le monimolimnion en dehors de la chemocline (soit entre 70 et $90 \mathrm{~m}$ de profondeur) une valeur de

$5.10^{-2} \mathrm{~m}^{2} \mathrm{j}^{-1}$, ce qui est en accord avec la valeur de $0,1 \mathrm{~m}^{2} \mathrm{j}^{-1}$ que nous avons choisie pour $85 \mathrm{~m}$.

Cette relation sera valable jusqu'à environ $55 \mathrm{~m}$ où une advection devient possible (présence de sources sous-lacustres vers $50 \mathrm{~m}$ de profondeur).

La figure 2 montre l'évolution du coefficient de diffusion turbulente en fonction de la profondeur. Comme ces variations sont très importantes, l'échelle utilisée est logarithmique.

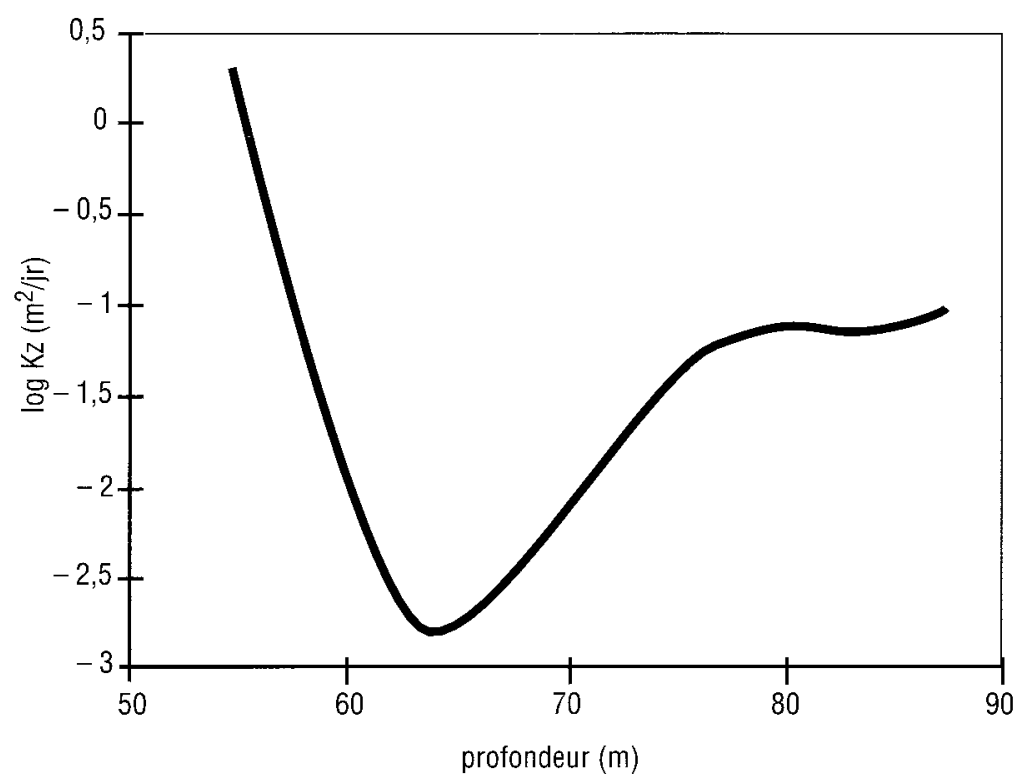

Figure 2 Coefficient de diffusion turbulente verticale (échelle logarithmique) en fonction de la profondeur.

Vertical eddy diffusion coefficient versus depth (logarithmic scale). 
Le coefficient $\mathrm{K}_{\mathrm{z}}$ est très faible au niveau de $63 \mathrm{~m}\left(\mathrm{~K}_{\mathrm{z}}=0,0017 \mathrm{~m}^{2} / \mathrm{j}\right)$ et nettement inférieur au coefficient de diffusion thermique moléculaire $\left(K_{\text {th }}=\right.$ $0,011 \mathrm{~m}^{2} / \mathrm{j}$; Carslaw and Jaeger, 1959). Les concentrations des éléments conservatifs et la température n'obéissent pas au même coefficient de diffusion. Bien que la température soit une quantité conservative, les relations concentration des conservatifs en fonction de la température ne sont pas linéaires, ce qui est effectivement observé dans le Pavin (VIOLLIER et al.,1995). La très faible valeur de $\mathrm{K}_{z}$ avait été notée par AESCHBACH-HERTIG et al. (2002), mais à défaut d'un échantillonnage suffisamment serré, ils n'avaient pu déterminer qu'une valeur moyenne dans ce qu'ils avaient appelé la chemocline (entre 60 et $68 \mathrm{~m}$ de profondeur) et qu'ils avaient chiffré à $0,0043 \mathrm{~m}^{2} / \mathrm{j}$.

\subsection{Estimation des vitesses de réaction}

Pour un élément réactif dont la concentration est notée $X$, la relation (1) devient :

$$
\frac{\partial X}{\partial t}=\frac{\partial}{\partial z}\left(K_{z} \cdot \frac{\partial X}{\partial z}\right)+R=0
$$

\subsubsection{Calcul général}

Les courbes « de mélange " $X=f(C)$ se prêtent relativement bien à une représentation polynomiale ; comme $C$ et $u$ sont liés par une relation linéaire, on cherchera à représenter $X$ en fonction d'un polynôme en $u$

$$
X(u)=\sum_{0}^{N} a_{n} \cdot u^{n}
$$

Considérons alors le terme général $a_{n} \cdot u^{n}$, et reportons-le dans l'équation (5)

Le calcul montre que

$$
\begin{gathered}
R_{X, n}=-n(n-1) \lambda a_{n} t^{n-2}[P(z)] \cdot\left[P^{\prime}(z) \operatorname{ch}^{-2}[P(z)]\right. \\
R_{X}=-\lambda P^{\prime}(z) \cdot \operatorname{ch}^{-2}[P(z)] \sum_{0}^{N} a_{n} n(n-1){ }^{*} t^{n-2}[P(z)]
\end{gathered}
$$

Cette expression montre, entre autres, que si la concentration de l'élément est constante $(\mathrm{N}=0)$ ou si elle est liée linéairement à $\mathrm{C}$ donc à $\mathrm{u}(\mathrm{N}=1)$, le terme réactionnel est nul, ce qui est pris en compte dans la définition des éléments conservatifs.

Nous avons représenté les concentrations des différents éléments en fonction de u par des polynômes de degré $n$ quand un polynôme de degré $n+1$ n'améliorait par le coefficient de corrélation $\mathrm{R}^{2}$.

\subsubsection{Rubidium, fer, vanadium, baryum}

Les concentrations de ces éléments peuvent être représentées en fonction de u par des polynômes. Le tableau 2 donne les valeurs de $a_{n}$ pour les différents éléments et les coefficients de corrélation correspondants. Les 
concentrations recalculées sont comparées aux concentrations mesurées sur les figures 3 à 6 .

Tableau 2 Coefficients des polynômes représentant les concentrations des éléments en fonction de $u$. Pour le manganèse les coefficients correspondent à l'équation (7).

Table 2 Coefficients of the polynomial representing element concentrations as a function of $u$. For manganese, the coefficients correspond to equation (7).

\begin{tabular}{lcccccc}
\hline & $\mathbf{R b}$ & $\mathbf{F e}^{*}$ & $\mathbf{V}$ & $\mathbf{B a}$ & & $\mathbf{M n}$ \\
\hline $\mathrm{a}_{0}$ & 180,92 & 350,16 & 49,62 & 18,505 & $\mathrm{a}_{0}$ & 17,5 \\
$\mathrm{a}_{1}$ & 76,52 & 579,24 & 40,226 & 34,871 & $\mathrm{a}_{1}$ & 17,5 \\
$\mathrm{a}_{2}$ & & 209,48 & $-73,101$ & 1,1867 & & \\
$\mathrm{a}_{3}$ & & & 40,14 & $-242,54$ & $\mathrm{~b}_{1}$ & 53 \\
$\mathrm{a}_{4}$ & & & 122,17 & $-45,836$ & & \\
$\mathrm{a}_{5}$ & & & & 644,82 & $\mathrm{z}^{*}$ & 0,698 \\
$\mathrm{a}_{6}$ & & & & 479,69 & & \\
$\mathrm{R}^{2}$ & 0,988 & 0,994 & 0,983 & 0,982 & & \\
\hline
\end{tabular}

* pour une profondeur supérieure à $62,8 \mathrm{~m}$.

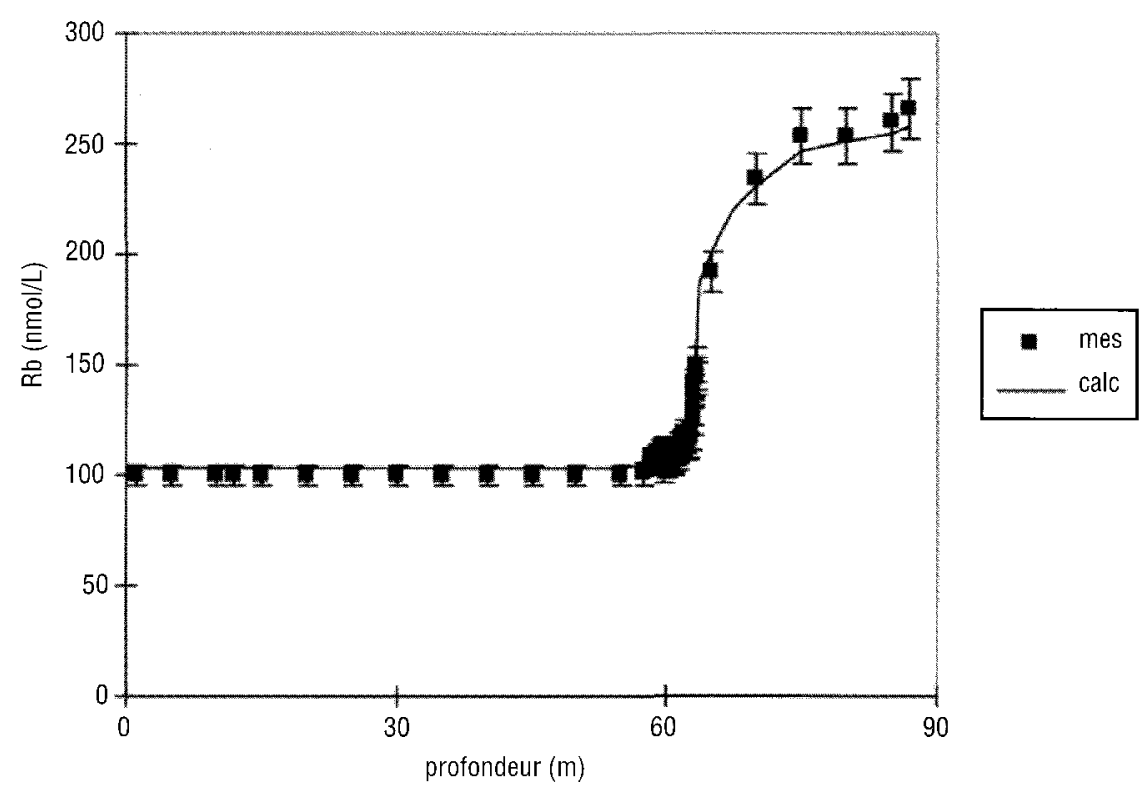

Figure 3 Concentration du rubidium dans le lac Pavin en fonction de la profondeur. Les carrés représentent les valeurs observées; la courbe en noir est la fonction calculée.

Rubidium concentration in lake Pavin versus depth. Squares are the observed concentrations; the black curve is the modeled concentrations. 

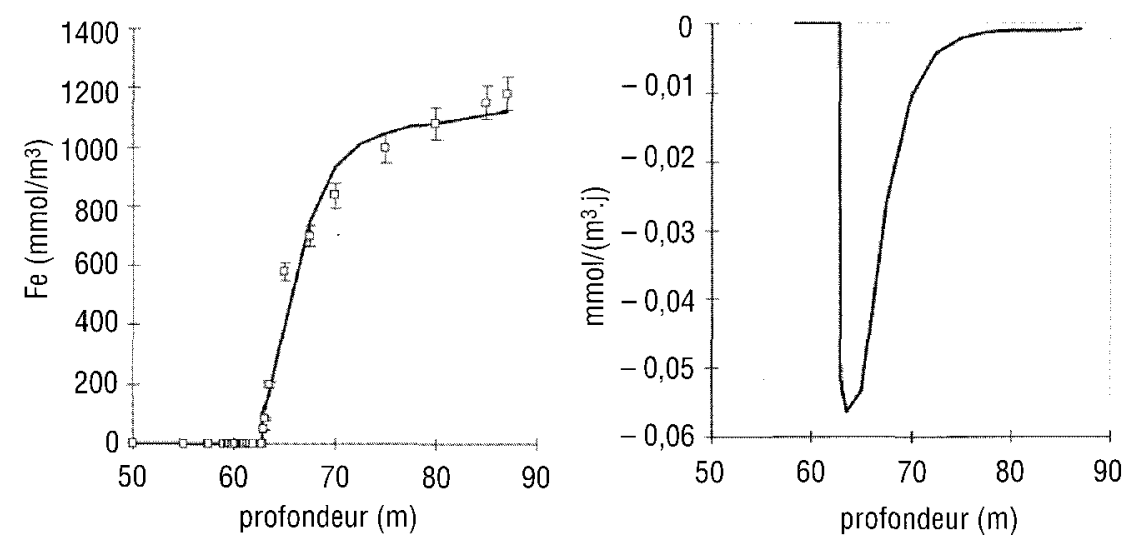

Figure 4 A gauche, concentration en fer en fonction de la profondeur (carrés : concentration observée ; courbe : concentration modélisée)

A droite, quantité de fer dissoute par $\mathrm{m}^{3}$ et par jour en fonction de la profondeur.

On the left, iron concentration versus depth (squares: observed concentrations; curve : modeled concentrations).

On the right, amount of dissolved iron per $\mathrm{m}^{3}$ and per day versus depth.
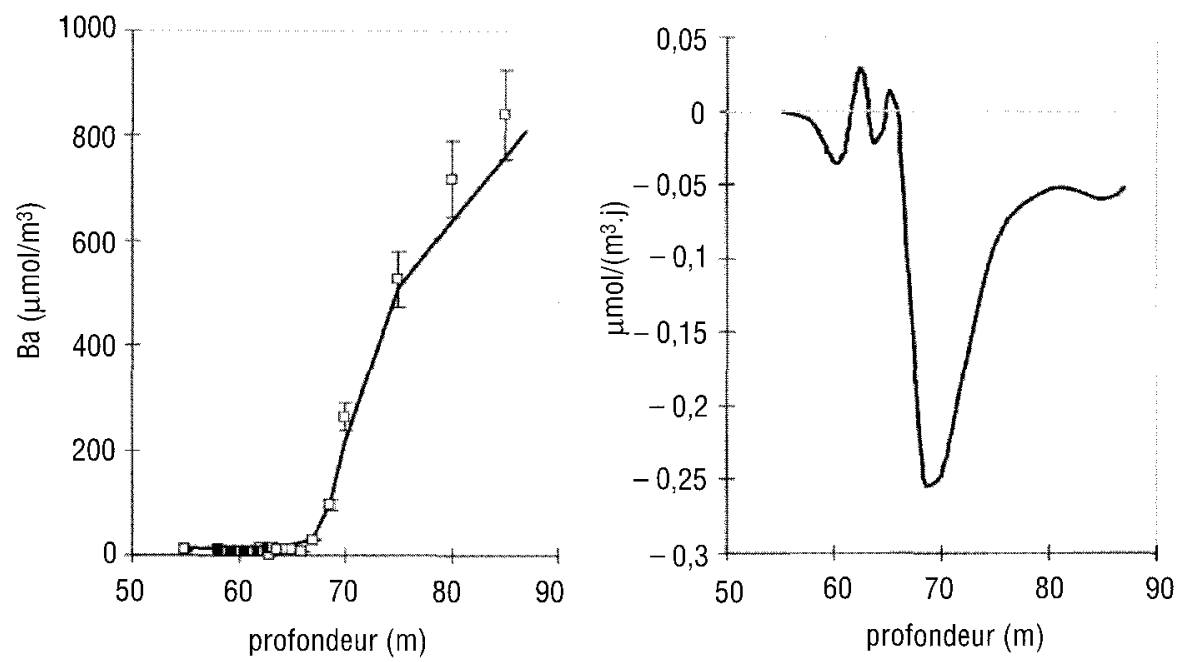

Figure 5 A gauche, concentration en baryum en fonction de la profondeur (carrés : concentration observée ; courbe : concentration modélisée).

A droite, quantité de baryum dissoute par $\mathrm{m}^{3}$ et par jour en fonction de la profondeur.

On the left, barium concentration versus depth (squares: observed concentrations ; curve : modeled concentrations).

On the right, amount of dissolved barium per $m^{3}$ and per day versus depth. 

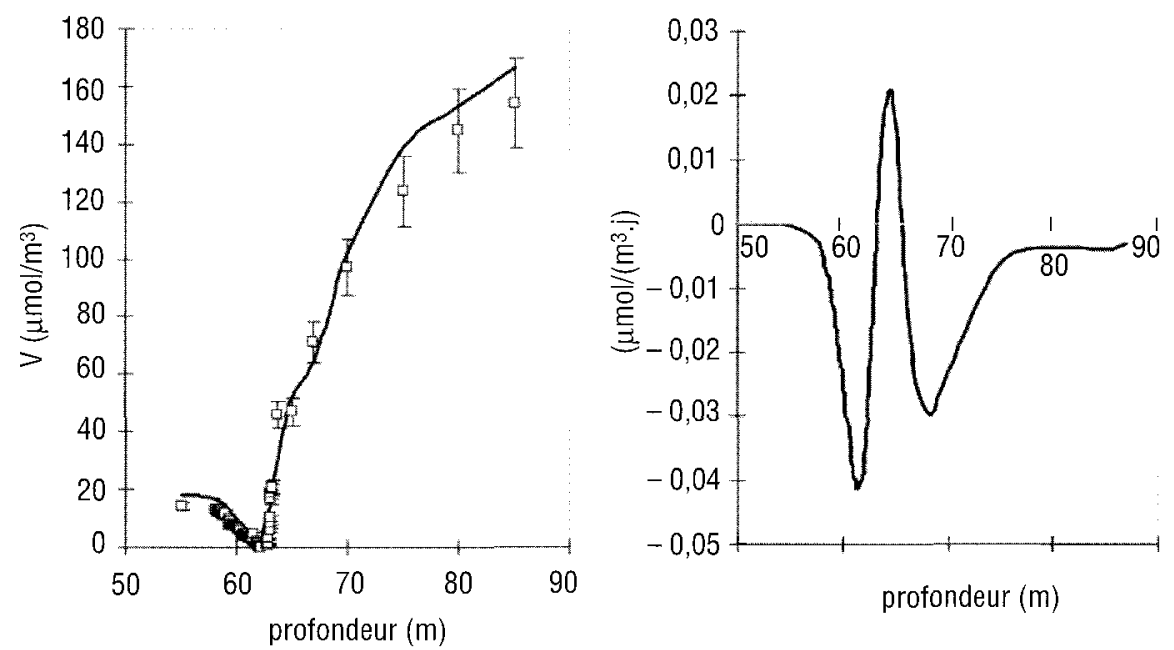

Figure 6 A gauche, concentration en vanadium en fonction de la profondeur (carrés : concentration observée ; courbe : concentration modélisée).

A droite, quantité de vanadium dissoute par $\mathrm{m}^{3}$ et par jour en fonction de la profondeur.

On the left, vanadium concentration versus depth (squares : observed concentrations ; curve : modeled concentrations).

On the right, amount of dissolved vanadium per $m^{3}$ and per day versus depth.

Les concentrations de rubidium sont liées linéairement à celles de sodium et à $u$. L'élément est donc conservatif et $R(R b)=0$ à toute profondeur.

Les concentrations de fer sont extrêmement faibles jusqu'à une profondeur de $63 \mathrm{~m}$ puis croissent très rapidement. Elles peuvent être représentées par une fonction du $2^{\mathrm{e}}$ degré en fonction de $u$.

La fonction représentant la dissolution, calculée en utilisant la formule (6) se réduit à un seul terme, est représentée sur la figure $4 \mathrm{~b}$. On voit qu'il y a précipitation dans toute la zone $55-85 \mathrm{~m}$ et que la vitesse de précipitation est maximale vers 63-64 m juste en dessous de l'IMM.

Comme pour les exemples suivants, la réaction de dissolution se produit au voisinage de l'interface eau-sédiment et devrait être étudiée à l'échelle du centimètre, voire du millimètre. Ceci n'est pas abordé dans cet article.

Les concentrations en baryum peuvent être représentées par un polynôme de degré 6 en fonction de $u$ avec un coefficient de corrélation satisfaisant. La courbe de dissolution est représentée sur la figure $5 b$. Il y a encore précipitation quelle que soit la profondeur, mais le maximum de précipitation se produit vers une profondeur de $70 \mathrm{~m}$.

Les concentrations de vanadium peuvent être représentées par un polynôme de degré 4 en fonction de $u$ avec un coefficient de corrélation satisfaisant. II y a précipitation en dessous de l'interface vers $63 \mathrm{~m}$; une faible 
dissolution à des profondeurs un peu supérieures et une précipitation importante vers $70 \mathrm{~m}$. Le vanadium suit donc vers $63 \mathrm{~m}$ le comportement du fer et vers $70 \mathrm{~m}$ celui du baryum.

\subsubsection{Manganèse}

Pour représenter la très forte augmentation de $\mathrm{Mn}$ au voisinage de l'interface nous avons choisi une fonction du type

$$
M n=a_{0}+a_{1} u+b_{1} \exp \left[-\left(z-z^{\circ}\right)^{2 / z^{*}}\right]
$$

Les coefficients correspondants figurent dans le tableau 2.

Les deux premiers termes ne correspondent à aucune réaction. II reste donc à calculer la réaction correspondant au $3^{e}$ terme en reprenant directement la formule (6). On voit qu'il y une très forte réactivité au voisinage de l'interface redox avec précipitation au dessus dans une zone à $\mathrm{K}_{\mathrm{z}}$ un peu plus élevé et une intense dissolution en dessous aux profondeurs de $\mathrm{K}_{\mathrm{z}}$ minimale.
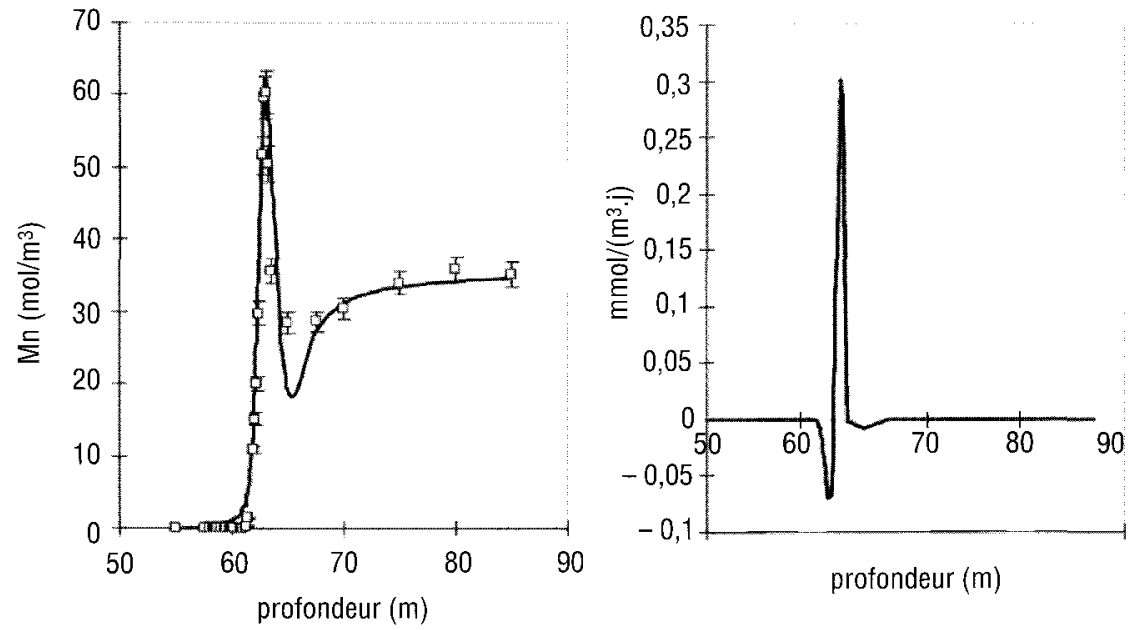

Figure $7 \quad$ A gauche, concentration en manganèse en fonction de la profondeur (carrés : concentration observée ; courbe : concentration modélisée).

A droite, quantité de manganèse dissoute par $\mathrm{m}^{3}$ et par jour en fonction de la profondeur.

On the left, manganese concentration versus depth (squares : observed concentrations; curve : modeled concentrations).

On the right, amount of dissolved manganese per $m^{3}$ and per day versus depth.

Contrairement aux éléments précédents, le manganèse semble peu concerné par les réactions sur le fond du lac. 


\subsection{Comparaison avec les flux particulaires}

En 1994-1995, Viollier et al. (1997) avaient mesuré les flux d'éléments à l'aide de trappes à sédiment disposées à 55,68 et $85 \mathrm{~m}$ de profondeur. Les trappes étaient relevées en moyenne tous les mois. Le tableau 3 rappelle les résultats concernant les 5 éléments.

Tableau 3 Flux descendants d'éléments sous forme particulaire (en $\mu \mathrm{mol}^{*} \mathrm{~m}^{-2 \star j} \mathrm{jr}^{-1}$ ) déduits de l'étude de Viollier et al. (1997).

Table 3 Downwards fluxes of particulate elements $\left(\mu \mathrm{mol}{ }^{*} \mathrm{~m}^{-2 *}\right.$ day $\left.{ }^{-1}\right)$ from Viollier et al. (1997).

\begin{tabular}{cccccc}
\hline Élément & $\mathbf{R b}$ & $\mathbf{F e}$ & $\mathbf{B a}$ & $\mathbf{V}$ & $\mathbf{M n}$ \\
\hline $55 \mathrm{~m}$ & 0,050 & 190 & 0,19 & 0,68 & 54,8 \\
$68 \mathrm{~m}$ & 0,057 & 440 & 0,27 & 1,48 & 7,4 \\
$85 \mathrm{~m}$ & 0,060 & 460 & 1,70 & 1,78 & 7,3 \\
\hline
\end{tabular}

Une comparaison quantitative est impossible car on ignore les vitesses de chute des particules solides. On peut néanmoins pour chaque élément localiser les profondeurs de réactions.

Comparativement aux autres éléments, les flux de rubidium sont très petits et l'augmentation absolue entre les 3 profondeurs est très faible ; ceci est en accord avec la faible réactivité de cet élément.

Les flux de fer sont nettement plus élevés à $68 \mathrm{~m}$ qu'à $55 \mathrm{~m}$, ce qui indique une importante précipitation entre ces 2 profondeurs ; la faible différence entre les flux à 68 et $85 \mathrm{~m}$ montrent que la précipitation de fer est moindre en dessous qu'au-dessus de $68 \mathrm{~m}$. Tout ceci est en accord avec les résultats de la présente étude (voir fig 4b).

Le flux de baryum particulaire est nettement plus important à $85 \mathrm{~m}$ qu'à $68 \mathrm{~m}$, ce qui montre que la précipitation de baryum se fait surtout en dessous de $68 \mathrm{~m}$.

Le flux de vanadium croît avec la profondeur, indiquant que la précipitation a lieu à la fois entre 55 et $68 \mathrm{~m}$ et entre 68 et $85 \mathrm{~m}$.

Enfin la très importante diminution du flux particulaire de manganèse entre 55 et $68 \mathrm{~m}$ montre que cet élément subit une dissolution entre ces 2 profondeurs.

Les résultats que l'on a obtenus ici confirment et permettent de préciser ce que VIOLLIER et al. (1997) avaient déduit de l'étude des trappes à sédiment. 


\section{5 - CONCLUSIONS}

Cette étude montre la très grande complexité des processus intéressant les éléments métalliques au voisinage de l'interface redox dans un lac. On peut distinguer 3 principales zones de réaction :

- le fond du lac (qui n'est pas abordé dans cette étude mais qui l'a été par exemple dans VIOLLIER et al. (1997)).

- la couche juste en dessous de l'interface redox

- une zone vers $70 \mathrm{~m}$ qui ne correspond pas à une limite précédemment identifiée.

En outre les réactions peuvent s'étaler sur un ou plusieurs mètres (précipitation de fer et du vanadium à $63 \mathrm{~m}$ ou précipitations à $70 \mathrm{~m}$ ) ou au contraire être très intenses et limitées à 12 ou $20 \mathrm{~cm}$ (précipitation ou dissolution du manganèse).

Seule une étude de tous les éléments majeurs (incluant des mesures de $\mathrm{pH}$ et $\mathrm{COD}$ ) à la même échelle de travail pourrait, avec l'aide de l'examen des phases solides, permettre d'identifier les processus qui entraînent ces variations.

\section{REMERCIEMENTS}

D. Lavergne et M. Evrard ont effectué les analyses d'éléments majeurs et M. Pèpe a contribué à celle des éléments trace. 


\section{RÉFÉRENCES BIBLIOGRAPHIQUES}

AESCHBACH-HERTIG W., HOFER M., KIPFER R. and IMBODEN D.M. (1999).Accumulation of mantle gases in a permanently stratified volcanic lake : lake Pavin, France. Geochim. Cosmochim. Acta, 63, 3357-3372.

AESCHBACH-HERTIG W., HOFER M., SCHMID M., KIPFER R. and IMBODEN D.M. (2002). The physical structure and dynamics of a deep meromictic lake (Lac Pavin ,France). Hydrobiologia, 487, 111136.

ALBÉRIC P., VIOLLIER E., JÉZÉQUEL D., GROSBOIS C. and MICHARD G. (2000). Interactions between trace elements and dissolved organic matter in the anoxic deep layer of Lake Pavin, Puy de Dôme, France. Limnol. Oceanog., 45, 10881096.

ALVINERIE J., DÉGOT B., LÉVĖQUE P. and VIGNEAUX M. (1966). Activité en tritium et caractéristiques chimiques des eaux du Lac Pavin - C. R. Acad. Sci., 262, 846-849.

AMBLARD C. and RESTITUITO F. (1984).Observations complémentaires en faveur de l'origine biogénique de la couche à hydrogène sulfuré d'un lac de moyenne montagne. C. R. Acad. Sci., 296, série II, 1787-1790.

BALISTRIERI L.S., MURRAY J.W. and PAUL B. (1992). The hydrogeochemical cycling of trace metals in the water column of lake Sammamish, Washington: response to seasonally anoxic conditions - Limnol. Oceanog., 37, 529-548.

BALISTRIERI L.S., MURRAY J.W. and PAUL B. (1994). The geochemical cycling of trace elements in a biogenic meromictic lake - Geochim. Cosmochim. Acta, 58, 19, 3993-4008.

BERNER R.A. (1964). An idealized model of dissolved sulfate distribution in recent marine sediments. Geochim. Cosmochim. Acta, 28, 1497-1503.

BERTHOULE A. (1896). Les lacs de Besse en Chandesse. Congrès Intern. Hydrol. Climatol. Geol. L'Auvergne, pp. 298-308.

BOYCE F.M. and HAMBLIN P.F. (1975).- A simple diffusion model of the mean field distribution of soluble materials in the
Great Lakes. Limnol. Oceanog., 20, 511517.

CAMUS G., MICHARD G., OLIVE Ph., BOIVIN P., DESGRANGES Ph., JÉZÉQUEL D., MEYBECK M., PEYRUS J.C., VINSON J.M., VIOLLIER E. and KORNPROBST J. (1993). Risque d'éruption gazeuse carbonique en Auvergne. Bull. Sci. Geol. Fr., 164, 767-78.

CARSLAW H.S. and JAEGER J.C. (1959). Conduction of heat in solids. Oxford, 510 p.

COSSA D., MASON R.P. and FITZGERALD W.F. (1994). Chemical speciation of mercury in a meromictic lake - in Mercury pollution, Integration and synthesis, Watras C.J. and Huckabee J.W. eds, Lewis publ., pp. 57-67.

DAVISON W. (1993). Iron and Manganese in lakes. Earth sci. Rev.,34, 119-163.

DELEBECQUE A. (1898). Les lacs français, 1 vol., Chamerot et Renouard (publ.), Paris

DEVAUX J., LAIR N. et AMBLARD C. (1983). Un écosystème lacustre profond : le lac Pavin. In Ecosystèmes limniques, Lamotte $M$. et Bourlière $P$. ed., Masson pp. 1-49.

DUSSART B. (1966). Limnologie. Masson. Paris.

GAILLARD J.-F. (1995). Limnologie chimique, in Pourriot R. et Meybeck M. Limnologie générale, pp.115-153, Masson, Paris.

HAMILTON Taylor J. and DAVISON W. (1995). Redox driven cycling of trace elements in lakes. In Physics and Chemistry of lakes, A. Lerman, D. Imboden and J. Gat ed, Springer, 217-264.

HESSLEIN R. (1976). An in situ sampler for close interval pore water studies. Limnol. Oceanog., 21, 912-914.

IMBODEN D. and WUEST A. (1995). Mixing mechanisms in lakes. In Physics and Chemistry of lakes, A. Lerman, D. Imboden and J. Gat ed., Springer, 83-138.

MARTIN J.M. (1985). The Pavin crater lake. In W. Stumm, ed, Chemical Processes in Lakes, Wiley New York, 169-188.

MARTIN J.M., MEYBECK M., NIJAMPURKAR V.N. and SOMAYAJULU B.L.K. (1992). 
${ }^{210} \mathrm{~Pb},{ }^{226} \mathrm{Ra}$ and ${ }^{32} \mathrm{Si}$ in Pavin lake. Chem. Geol., 94, 173-181.

MEYBECK M., MARTIN J.M. and OLIVE P. (1975). Géochimie des eaux et des sédiments de quelques lacs volcaniques du Massif Central - Vehr. Int. Ver. Limnol., 19, 1150-1165.

MICHARD G. (1971). Theoretical model for manganese distribution in calcareous sediment cores. J. Geophys. Res., 76, 2179-2186.

MICHARD G. (2002). Chimie des eaux naturelles, Ed. Publisud, Paris $480 \mathrm{p}$.

MICHARD G., VIOLLIER E., JÉZÉQUEL D. and SARAZIN G. (1994). Geochemical study of a crater lake. The Pavin lake, France : Identification, location and quantification of the chemical reactions in the lake-Chem. Geol. 115, 103-115.

MICHARD G., JÉZÉQUEL D., PRÉVOT F., SARAZIN G., VIOLLIER E. and ALBÉRIC P. (2002). Biogeochemical processes in Lake Pavin. Hydrobiologia, Special Issue (sous presse).

MORTIMER C.H. (1941). The exchange of dissolved substances between mud and water in lakes. (Parts I and II). J. Ecol., $29,280-329$.

OLIVIER L. (1952). Note sur la présence en été, dans le lac Pavin, d'une couche dépourvue d'oxygène vers $70 \mathrm{~m}$ de profondeur. C. R. Acad. Sci. Paris, 234, 743-745.

PELLETIER J.P. (1968). Un lac méromictique : le Pavin (Auvergne), Ann. Stat. Biol. Besse en Chandesse, 3, 147.

RABOUILLE C. et GAILLARD J.-F. (1991). Toward the EDGE : Early diagenetic global explanation : a model depicting the early diagenesis of organic matter, $\mathrm{O}_{2}$, $\mathrm{NO}_{3}^{-}, \mathrm{Mn}$ and $\mathrm{PO}_{4}$, Geochim. Cosmochim. Acta, 55, 2511-2525.

RESTITUITO F. (1984). Contribution à l'étude du sédiment d'un lac oligomésotrophe d'origine volcanique (le lac Pavin, France) Hydrobiol., 109, 229-234.

RESTITUITO F. (1987). Consequences of redox conditions on the distribution of cations in a meromictic oligotrophic lake. Hydrobiol., 144, 63-75.

SEYLER P. and MARTIN J.M. (1989). Biogeochemical processes affecting arsenic species distribution in a permanently stratified lake. Environ. Sci. Technol., 23, 1258-1263.

SUGIYAMA M., HORI T., KIHARA S. and MATSUI M. (1992). A geochemical study of the specific distribution of barium in Lake Biwa, Japan. Geochim. Cosmochim. Acta, 56, 597-605.

TAILLEFERT M., MACGREGOR B., GAILLARD J.F., LIENEMANN C.P., PERRET D. and STAHL D.A. (2002). Evidence for a dynamic cycle between $\mathrm{Mn}$ and $\mathrm{Co}$ in the water of a stratified lake. Envir. Sci. Techn., 36, 468-476.

VAN CAPPELLEN Ph. and WANG Y. (1996). Cycling of iron and manganese in surface sediments : a general theory for the coupled transport and reaction od carbon, oxygen, nitrogen, sulfur, iron and manganese. Am. J. Sci, 296, 197-243.

VIOLLIER E. (1995). Géochimie des éléments traces en milieu lacustre. Thèse Université Paris 7, $306 \mathrm{p}$.

VIOLLIER E., ALBÉRIC P., JÉZÉQUEL D., MICHARD G., PËPE $M$. and SARAZIN G. (1995). Geochemical study of a crater lake, the Pavin lake, France. Trace element behavior in the monimolimnion. Chem Geol., 125, 61-72.

VIOLLIER E., JÉZÉQUEL D, MICHARD G., PĖPE M. and SARAZIN G. (1997). Geochemical study of a crater lake : lake Pavin, Puy de Dôme, France. Constraints afforded by the particulate matter distribution in the element cycling within the lake. Chem. Geol., 142, 225241.

WANG Y. and VAN CAPPELLEN Ph. (1996). A multicomponent reactive transport model of early diagenesis : application in redox cycling in coastal marine sediments. Geochim. Cosmochim. Acta, 60, 2993-3014.

WETZEL R.G., (1983). Limnology, Saunders college, Philadelphie, 766 p. 